\title{
A giant neurothekeoma of the left shoulder blade: A case report
}

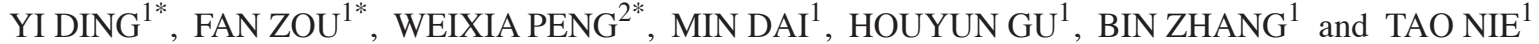 \\ ${ }^{1}$ Department of Orthopedics, The First Affiliated Hospital of Nanchang University, Nanchang, Jiangxi 330006; \\ ${ }^{2}$ Department of Administration, The Fourth Affiliated Hospital of Nanchang University, Nanchang, Jiangxi 330003, P.R. China
}

Received November 12, 2014; Accepted September 17, 2015

DOI: $10.3892 / \mathrm{ol} .2015 .4028$

\begin{abstract}
Neurothekeoma is a rare myxoma of the peripheral nerve sheath. The current report presents a case of a giant neurothekeoma with a partially-formed capsule, scapula erosion and unclear biological behavior, which originated in the intermuscular space between the left trapezius muscle and scapula. The patient was initially misdiagnosed with a fibromatosis using computed tomography and magnetic resonance image scanning. Diagnosis of the neoplasm was confirmed by pathological and immunohistochemical examination, revealing a neurothekeoma with unclear biological behavior. The patient underwent a wide and complete local resection. Using a comprehensive postoperative follow-up strategy, it was determined that the patient recovered well. The tumor was $\sim 17 \times 16 \times 10 \mathrm{~cm}$ in size and was in contact with the scapula. The purpose of the present study was to describe a rare giant neurothekeoma and review the diagnostic techniques utilized to reach a definitive diagnosis. Histopathological and immunohistochemical analyses were recommended for the diagnosis of neurothekeoma. There have been no previous reports regarding neurothekeomas exhibiting malignant transformation. Early and complete surgical resection is considered to be an effective method of treating this type of neurothekeoma.
\end{abstract}

\section{Introduction}

Neurothekeoma is a rare and typically benign cutaneous tumor. Neurothekeoma possesses a distinctive histological appearance and characteristic clinical features, and is thought to be a variant of a peripheral nerve sheath myxoma (1-3). Neurothekeomas are generally slow-growing and manifest as a solitary papule or nodule, which is typically located on the head, neck or upper extremities (4-10). In a small number of cases, the

Correspondence to: Professor Tao Nie, Department of Orthopedics, The First Affiliated Hospital of Nanchang University, 17 Yongwai Main Street, Nanchang, Jiangxi 330006, P.R. China E-mail: 413097263@qq.com

*Contributed equally

Key words: neurothekeoma, surgery, shoulder blade, misdiagnosis, fibromatosis solitary papule or nodule may be located in the oral cavity, breast, tongue, maxilla (11-14), cranial cavity (10) or spinal intradural space (15). Though the shoulder has been described as a common site of neurothekeoma development (3-6), there have been few cases of humeral neurothekeoma reported in detail in the relevant literature $(6,16)$. Tumors such as that reported in the present case study remain a rare occurrence. The current study aimed to introduce a rare giant neurothekeoma that developed in the left should blade of an elderly man over $>10$ years. The present neurothekeoma originated from the intermuscular space of the left shoulder blade, and presented with a partially-formed capsule, scapula erosion and unclear biological behavior. The tumor was $\sim 17 \times 16 \times 10 \mathrm{~cm}$ in size, and occurred in an 81-year-old man with a $>10$ year medical history of a slow-growing mass.

Initially, the patient was incorrectly diagnosed with fibromatosis, based on the characteristic clinical symptoms and imageological diagnosis. Results of computed tomography (CT) and magnetic resonance imaging (MRI) scans indicated a diagnosis of fibromatosis. However, immunohistochemical and pathological examination of the lesion suggested a diagnosis of a neurothekeoma originating in the peripheral nerve sheath. Clinical examination and patient history are significant in the diagnosis of disease, however immunohistochemical staining and pathological sectioning are the standard methods of diagnosis for neurothekeoma $(1-3,17,18)$. In order to correctly guide treatment, definitive preoperative diagnosis of neurothekeoma is of significance. The patient was treated with a wide local excision, performed by professional bone tumor surgeons. Written informed consent was obtained from the patient.

\section{Case report}

An 81-year-old man was admitted to the Department of Orthopedic Oncology of the First Affiliated Hospital of Nanchang University (Nanchang, China), and presented with a slow-growing, painless mass that had developed over a period of 10 years, in the left shoulder blade. In the previous 3 years, the tumor had grown significantly more rapidly than in the preceding 10 years. The principal clinical manifestation of the tumor was numbness of the left hand, which was not mitigated by rest. Physical examination of the patient revealed a giant mass on the inner side of the left humeral back, which was immobile, tender and exhibited distinct borders (Fig. 1A). The large mass prevented the shoulder moving in all directions 

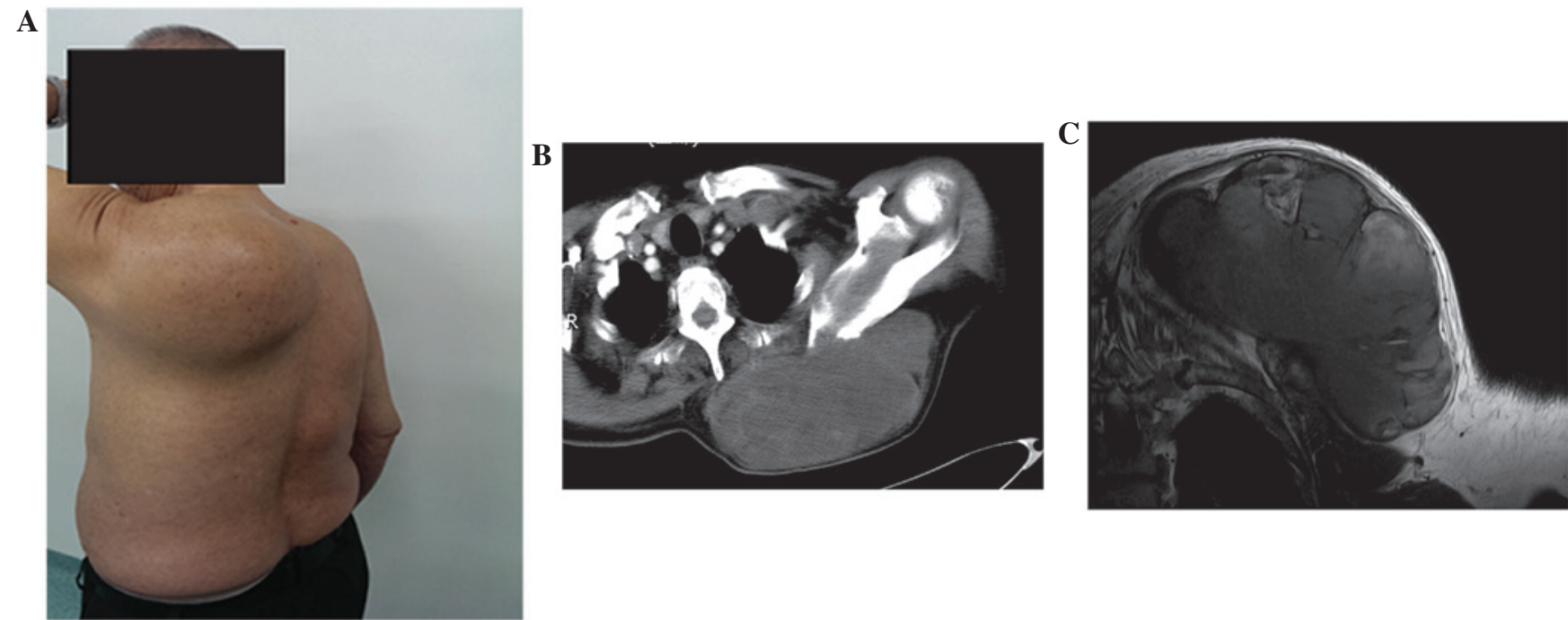

Figure 1. Preoperative observations. (A) The primary clinical feature was a painless giant mass located on the left humeral back. (B) Computed tomography scanning revealed a giant mass with homogeneous density and no bone destruction, $\sim 17 \times 16 \times 10 \mathrm{~cm}$ in size. (C) Magnetic resonance imaging scanning revealed that the mass exhibited multiple linear low signal intensity strands on the T1 weighted image, with well-defined margins.

and passive activities were limited. No abnormalities were identified during the medical examination, with all results of routine laboratory tests, such as erythrocyte sedimentation rate, within the normal ranges. The patient exhibited a prior history of hypertension, and blood pressure had been controlled using antihypertensive drug treatment $(30 \mathrm{mg}$ nifdipine controlled-release tablets, p.o., q.d.).

CT scanning revealed a large lobulated and cystic-solid mass, $\sim 17 \times 16 \times 10 \mathrm{~cm}$ in size, exhibiting homogeneous density and a clear border below the left trapezius muscle (Fig. 1B). Bone hyperplasia hardening of the left scapula was also revealed using $\mathrm{CT}$, however, no bone destruction was observed. Based on the CT scanning results, borderline or poorly differentiated malignant fibromatosis was diagnosed by a professional radiologist.

In order to achieve further confirmatory diagnosis, MRI scanning was performed (Fig. 1C), which revealed a giant and lobulated lump, measuring $\sim 17 \times 16 \times 10 \mathrm{~cm}$ in size. MRI scanning of the mass revealed miscellaneous signals, primarily including long intensity for T1 and T2 signals. The tumor was observed as multiple linear low signal intensity strands, and the tumor border was well-defined. The soft tissue surrounding the mass demonstrated normal signal intensity. No obvious destructive signal intensity in the left scapula was identified. Following MRI scanning, no enlarged lymph nodes or distant metastases were identified. MRI scanning also suggested a potential diagnosis of fibromatosis. However, the tumor exhibited unclear biological behavior, with no examinations confirming whether the tumor was benign, borderline or malignant.

The tumor resection was performed by professional surgeons. Following the administration of general anesthesia (6 $\mathrm{mg} / \mathrm{kg} / \mathrm{h}$ propofol intravenous drip), the operative site was disinfected with an iodophor three times, and routine sterile drapes were placed in the right lateral position in order to avoid contamination and expose the operative field. A spindle-shaped surgical incision, $26 \mathrm{~cm}$ in length, was made in the center of the left humeral mass. Subsequently, the surgeons separated the skin, subcutaneous superficial fascia and left trapezius muscle in order to isolate the tumor, which was almost entirely surrounded by a soft tissue capsule. However, the tumor face adjacent to the left scapula possessed an area of $\sim 3 \times 4 \mathrm{~cm}$ without a capsule. Notably, bone destruction of the left scapula tumor interface was revealed following removal of the mass. An osteotome and rongeur were utilized to resect the destroyed bone and residual tumor tissue, until complete excision was achieved. Pathological examination of the excised mass was subsequently performed.

The excised specimen was off-white in color, and was measured to be $\sim 17 \times 16 \times 10 \mathrm{~cm}$ (Fig. 2A). The tumor surface was smooth and clear, however there was an area of $\sim 3 \times 4 \mathrm{~cm}$ on the scapula-tumor interface from which capsule was absent. Microscopic evaluation of hematoxylin and eosin (H\&E) stained slides (Fig. 2B and C) revealed that the tumor possessed myxoid lobulated lesions. The tumor was encapsulated by a thin fibrous connective tissue and was composed of ovoid lobules, separated by fibrous septae and arranged in well-formed micronodules. The lobules were formed of loosely arranged stellate and spindle-shaped cells. Necrosis and mitosis were almost absent. Immunohistochemical staining revealed that the tumor cells were positive for S100 and negative for desmin, cluster of differentiation 34 and smooth muscle actin (Fig. 3). Based on these histopathological results, the present case was diagnosed as neurothekeoma. The patient demonstrated no evidence of tumor recurrence for 3 years subsequent to the performance of surgery.

\section{Discussion}

To the best of our knowledge, neurothekeoma is an uncommon and benign dermal tumor, originating from the sheath of peripheral nerves (19). Harkin and Reed (20) initially reported neurothekeoma in 1969, as a rare neoplasm arising in the endoneurium of peripheral nerves, characterized by 
A

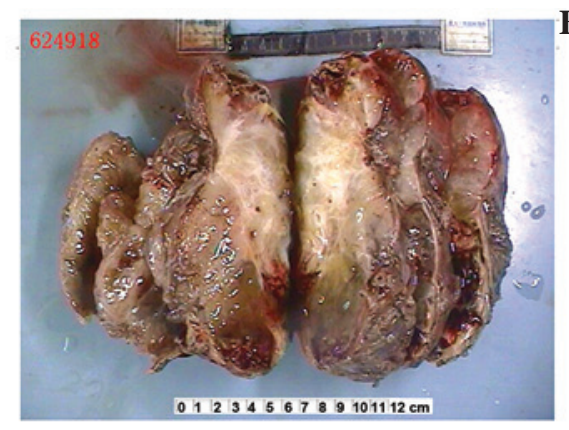

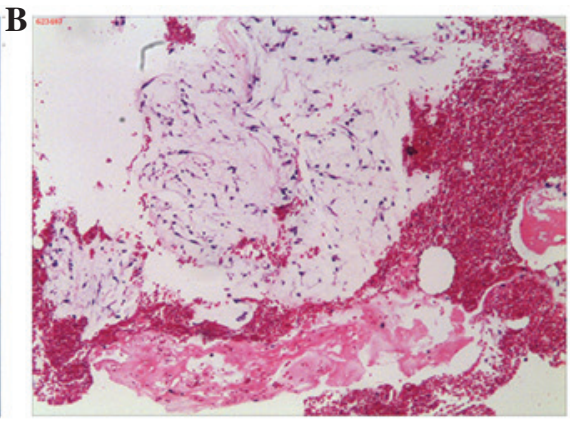

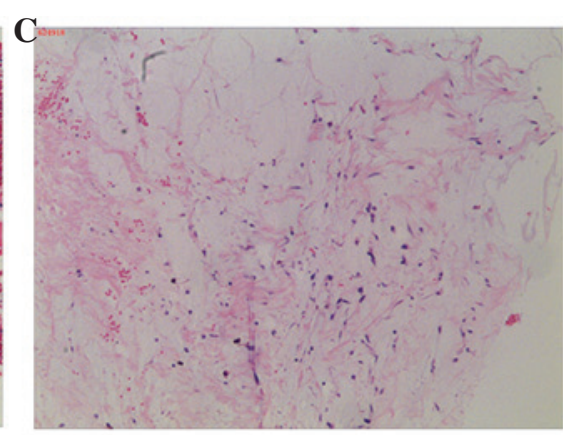

Figure 2. Histological observations. (A) The excised specimen was off-white in color and $7 \times 16 \times 10 \mathrm{~cm}$ in size. (B) Histopathological examination of H\&E-stained sections revealed a mucinous mass a with lobular arrangement (magnification, x100). (C) The lobules in the mass were comprised of loosely arranged cells, though focal areas revealed closely clustered thin-spindled and stellate cells (H\&E staining; magnification, x100). H\&E, hematoxylin and eosin.

A

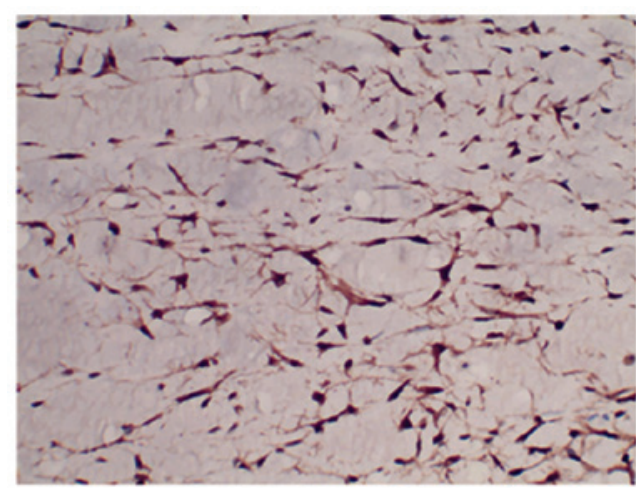

C

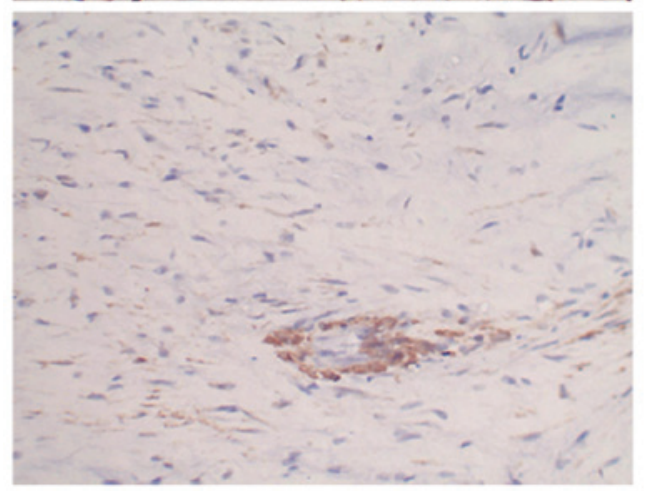

B

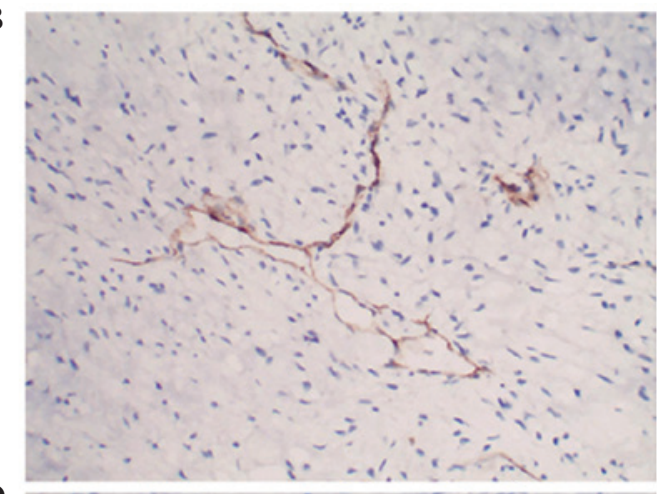

D

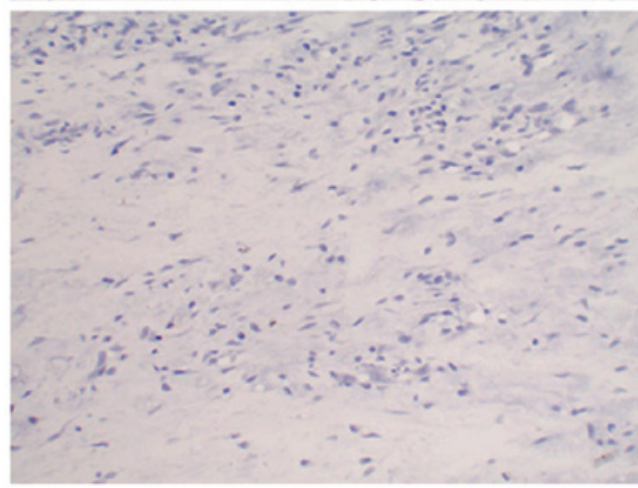

Figure 3. Immunohistochemical staining revealed that the tumor cells were positive for (A) S100. However, tumor cells were negative for (B) cluster of differentiation 34, (C) smooth muscle actin and (D) desmin (magnification, x200).

an abundant mucoid matrix; and classed it as a myxoma of the nerve sheath. Gallager and Helwig initially suggested the terminology of neurothekeoma in 1980 (3). Based on Papadopoulos et al (19), who performed a study of the largest group of neurothekeoma cases $(\mathrm{n}=292)$ to the best of our knowledge, it may be concluded that the most common site of neurothekeoma occurrence is the upper extremities (33.6\%), followed by the head and neck (29.4\%), trunk (17.2\%), lower extremities $(9.7 \%)$ and mucosal membranes $(9.3 \%)$. A markedly lower number of neurothekeomas $(\sim 0.8 \%)$ were located in the spinal marrow (19). Alexandru et al (10) reported the case of a neurothekeoma identified in the posterior fossa. Neurothekeomas have been identified in patients ranging in age from 15 months to 84 years, with a mean age of 28 years, and the lesions are most commonly identified in patients aged between 10 and 30 years old $(19,21)$. Neurothekeomas remain uncommon in patients $>80$ years of age $(19,21)$. The incidence of neurothekeomas is 2-fold greater in women, compared with that of men (19). Papadopoulos et al (19) additionally reported that the average diameter of a neurothekeoma was $1.2 \mathrm{~cm}$.

In the present case, an 81-year-old man with a $>10$ year clinical history of a slow-growing mass, was diagnosed with neurothekeoma via immunohistochemical and pathological examination. CT and MRI scanning identified the neoplasm between the left trapezius muscle and scapula. Neurothekeomas are typically asymptomatic and slow growing $(19,22)$. However, in the present case, the mass had grown considerably more rapidly in the most recent 3 years, compared with the preceding 10 years, and the patient experienced numbness in the left hand, which was not mitigated by rest. Based on the unique clinical characteristics and large size of the tumor, to the best of our knowledge, the present case report is the first description of a giant neurothekeoma with unclear biological behavior, which originated in the intermuscular space. 
Previous studies have reported that neurothekeomas possessing spindle or stellate cells, embedded in an abundant myxoid background, may be classified as classical, cellular and mixed types, based on their cellularity, mucin content and growth pattern, or the quantity of myxoid matrix $(5,23,24)$. However, certain scientists do not support the current classification of neurothekeoma, due to the lack of immunohistochemical and ultrastructural evidence to support a nerve sheath origin $(9,25)$. Fetsch et al (5) reported that the term neurothekeoma was used to describe cellular and mixed tumor variants, and that the term nerve sheath myxoma was used for lesions. According to previous studies, immunohistochemical markers, including S100 protein, glial fibrillary acidic protein, nerve growth factor receptor, cluster of differentiation 57, NKI/C3, Ki-M1p, and cluster of differentiation 68, may be used in order to distinguish between the 3 subtypes of neurothekeoma $(4,5,11,19,26-28)$. In addition, Sheth et al (29) initially used the molecular technique of gene expression profiling to evaluate the hypothesis that dermal nerve sheath myxomas are of peripheral nerve sheath origin, and suggested that neurothekeomas may be a variant of fibrous histiocytomas. There was no definite somatotype of neurothekeoma or nerve sheath myxoma identified in the present study. To clarify the somatotype, immunohistochemical marker testing and gene expression profiling technology may be useful.

The clinical differential diagnosis of neurothekeoma is multitudinous, including schwannoma, true neuroma, myxoid neurofibroma, ossifying fibromyxoid tumor, myxoid malignant fibrous histiocytoma, melanocytic lesions and epithelioid hemangioma $(19,24,30)$. In order to perform a definitive diagnosis of neurothekeoma, a full review, including clinical manifestation, careful physical and imageological (CT and MRI) examination, immunohistochemical staining and pathological sectioning, should be taken into consideration. Furthermore, immunohistochemical marker testing and gene expression profiling technology may be utilized in order to clarify the tumor somatotype.

The current patient was diagnosed with a neurothekeoma, following discussion by the pathologists in the First Affiliated Hospital of Nanchang University. Due to the fact that incomplete excision of the lesion may lead to local recurrence $(5,17)$, surgical resection was performed in order to minimize the risk of tumor relapse. Neurothekeoma is a rare benign tumor and, to the best of our knowledge, there have been no reported cases of malignant transformation (31), therefore treatment with chemotherapy and radiotherapy is not required. However, the neoplasm presented with a partially-formed capsule, scapula erosion and unclear biological behavior, which indicated the potential for malignant transformation. A comprehensive follow-up strategy was conceived by the professional bone tumor surgeons, and used to confirm that the patient recovered well following surgical resection of the tumor.

In conclusion, the present case study described a rare giant neurothekeoma, which was identified in the intermuscular space of the left shoulder blade. The mass was painless and had been slowly growing for $>10$ years. Immunohistochemical and pathological observations allowed the achievement of a definitive diagnosis, whereas initial imageological examinations resulted in a false diagnosis. Therefore, diagnostic pathology and immunostaining is necessary for the diagnosis of a neurothekeoma. Due to the possibility of malignant transformation, complete excision is recommended for the treatment of neurothekeomas of this size. Follow-up was accomplished and the patient has recovered, and demonstrated no evidence of tumor recurrence for 3 years subsequent to surgery.

\section{References}

1. Isoda M and Katayama M: Neurothekeoma. Cutis 41: 255-256, 1988.

2. Aronson PJ, Fretzin DF and Potter BS: Neurothekeoma of Gallager and Helwig (dermal nerve sheath myxoma variant): Report of a case with electron microscopic and immunohistochemical studies. J Cutan Pathol 12: 506-519, 1985.

3. Gallager RL and Helwig EB: Neurothekeoma - a benign cutaneous tumor of neural origin. Am J Clin Pathol 74: 759-764, 1980.

4. Yun SJ, Park HS, Lee JB, Kim SJ, Lee SC and Won YH: Myxoid cellular neurothekeoma: A new entity of S100-negative, CD68-positive myxoid neurothekeoma. Ann Dermatol 26: 510-513, 2014

5. Fetsch JF, Laskin WB, Hallman JR, Lupton GP and Miettinen M: Neurothekeoma: An analysis of 178 tumors with detailed immunohistochemical data and long-term patient follow-up information. Am J Surg Pathol 31: 1103-1114, 2007.

6. Yang YW, Shih IH, Huang YH, Kuo TT and Hong HS: Mixed-type neurothekeoma presenting with an unusual clinical appearance of multiple satellite lesions on the back. Dermatol Surg 31: 720-722, 2005.

7. Oh SH, Lee HJ, Chang SE, Lee MW, Choi JH, Moon KC and Koh JK: A case of cellular neurothekeoma. Korean J Dermatol 44: 1126-1129, 2006

8. Ryu DJ, Kim HJ, Jung JY, Kwon YS and Lee JH: A case of myxoid neurothekeoma on the hand. Korean J Dermatol 47: 982-985, 2009.

9. Fetsch JF, Laskin WB and Miettinen M: Nerve sheath myxoma: A clinicopathologic and immunohistochemical analysis of 57 morphologically distinctive, S-100 protein- and GFAP-positive, myxoid peripheral nerve sheath tumors with a predilection for the extremities and a high local recurrence rate. Am J Surg Pathol 29: 1615-1624, 2005.

10. Alexandru D, Satyadev R and So W: Neurothekeoma in the posterior fossa: Case report and literature review. Perm J 16: 63-64, 2012.

11. Vered M, Fridman E, Carpenter WM and Buchner A: Classic neurothekeoma (nerve sheath myxoma) and cellular neurothekeoma of the oral mucosa: Immunohistochemical profiles. J Oral Pathol Med 40: 174-180, 2011.

12. Wee A, Tan CE and Raju GC: Nerve sheath myxoma of the breast. A light and electron microscopic, histochemical and immunohistochemical study. Virchows Arch A Pathol Anat Histopathol 416: 163-167, 1989.

13. Makino T, Utsunomiya T, Kamino Y, Kobayashi R, Fukumoto M, Yamamoto $\mathrm{H}$ and Nagura $\mathrm{H}$ : Nerve sheath myxoma of the tongue in a child. Int J Oral Maxillofac Surg 31: 451-454, 2002.

14. Cohen NA, Samadi DS, Pawel BR and Kazahaya K: Cellular neurothekeoma of the maxilla. Ann Otol Rhinol Laryngol 113: 384-387, 2004

15. Paulus W, Jellinger $\mathrm{K}$ and Perneczky G: Intraspinal neurothekeoma (nerve sheath myxoma). A report of two cases. Am J Clin Pathol 95: 511-516, 1991.

16. Woo EK, Lim TK and Tan SH: Neurothekeomas of the upper limb - case series and clinicopathological review. Hand Surg 10: 311-317, 2005.

17. Wiemeyer S and Hafer G: Neurothekeoma of the toe. Foot Ankle Spec 6: 479-481, 2013.

18. López-Cepeda LD, Navarrete-Franco G, Novales-Santacoloma J and Enriquez-Merino J: Scar-like lesion on dorsal nose (cellular neurothekeoma). Head Face Med 3: 39, 2007.

19. Papadopoulos EJ, Cohen PR and Hebert AA: Neurothekeoma: Report of a case in an infant and review of the literature. J Am Acad Dermatol 50: 129-134, 2004.

20. Harkin JC and Reed RJ: Solitary benign nerve sheath tumors, myxoma of the nerve sheath. In: Tumors of the Peripheral Nervous System, Atlas of Tumor Pathology, Second Series, Fascicle 3. Harkin JC and Reed RJ (eds). Armed Forces Institute of Pathology, Washington, DC, pp60-64, 1969. 
21. Seo BF, Kang HW, Lee JY, Kwon H and Jung SN: Ankle neurothekeoma: A case report. J Foot Ankle Surg 52: 678-680, 2013.

22. Rawal YB, Mustiful-Martin D, Rosebush MS, Anderson KM and Mincer HH: Slow-growing gingival mass. Oral Surg Oral Med Oral Pathol Oral Radiol 113: 161-167, 2012.

23. Argenyi ZB, LeBoit PE, Santa Cruz D, Swanson PE and Kutzner H: Nerve sheath myxoma (neurothekeoma) of the skin Light microscopic and immunohistochemical reappraisal of the cellular variant. J Cutan Pathol 20: 294-303, 1993.

24. Nishioka M, Aguirre RL, Ishikawa A, Nagumo K, Wang LH and Okada N: Nerve sheath myxoma (neurothekeoma) arising in the oral cavity: Histological and immunohistochemical features of 3 cases. Oral Surg Oral Med Oral Pathol Oral Radiol Endod 107: e28-e33, 2009.

25. Laskin WB, Fetsch JF and Miettinen M: The 'neurothekeoma': Immunohistochemical analysis distinguishes the true nerve sheath myxoma from its mimics. Hum Pathol 31: 1230-1241, 2000.

26. Chang SE, Lee TJ, Ro JY, Choi JH, Sung KJ, Moon KC and Koh JK: Cellular neurothekeoma with possible neuroendocrine differentiation. J Dermatol 26: 363-367, 1999.
27. Husain S, Silvers DN, Halperin AJ and McNutt NS: Histologic spectrum of neurothekeoma and the value of immunoperoxidase staining for S-100 protein in distinguishing it from melanoma. Am J Dermatopathol 16: 496-503, 1994.

28. Mahalingam M, Alter JN and Bhawan J: Multiple cellular neurothekeomas - a case report and review on the role of immunohistochemistry as a histologic adjunct. J Cutan Pathol 33: 51-56, 2006.

29. Sheth S, Li X, Binder S and Dry SM: Differential gene expression profiles of neurothekeomas and nerve sheath myxomas by microarray analysis. Mod Pathol 24: 343-354, 2011.

30. Tiffee JC and Pulitzer DR: Nerve sheath myxoma of the oral cavity: Case report and review. Oral Surg Oral Med Oral Pathol Oral Radiol Endod 82: 423-425, 1996.

31. Kah TA, Yong KC and Annuar FH: Neurothekeoma palpebrae in association with multiple superficial angiomyxomas: Tegumental angiomyxoma-neurothekeoma syndrome (TAN syndrome). Clin Pract 1: e67, 2011. 